\title{
A Greedy Optimization Algorithm Based on QoS in UAV Cluster Network
}

\author{
Wei $\mathrm{Fu}^{1, \text { a) }}$, Xinli Zhou ${ }^{2}$ \\ ${ }^{1}$ School of Coast Defense, Naval Aeronautical University, Yantai 264001, China \\ ${ }^{2}$ School of Aviation Operations Service, Naval Aeronautical University, Yantai 264001, China. \\ a)1119219753@qq.com
}

\begin{abstract}
Due to the strong mobility of nodes, the possibility and randomness of network holes were increasing in UAV network, and the limited energy-carrying capacity of nodes leads to the limitation of cruising ability. The general geographic assistant routing protocols often failed to satisfy demand in the network. A greedy optimization algorithm based on Quality of Service (QoS) was proposed in this paper. The mechanism set segment points based on the route forwarding strategy, in order to optimize the detour path part, and guarantees to response the existing network holes or optimize a new network hole duly. Considering the actual situation of the stability of the UAV communication links and the limited energy-carrying capacity, comprehensive criteria based on QoS was provided for selection of nodes according to the actual application requirements. The simulation shows that compared with the GPSR algorithm and BOPF algorithm the algorithm can optimize the transmission path, reduce end-to-end delay, and improve the performance of the network.
\end{abstract}

Key words: UAV network, routing hole, Quality of Service, node criterion.

\section{INTRODUCTION}

The current combat mode of UAV has changed from a stand-alone operation to a multi-aircraft joint operation. The full range of combat capabilities exhibited by the UAV group is unmatched by a single one, but this kind of high-speed and high-dynamic combat environment needs more flexible communication methods and strict standards. Mobile Ad Hoc Network (MANET) has become the preferred method for cluster operations because of its characteristics of no center, self-organization, dynamic topology and multi-path. Although this ad hoc network has many advantages over other networking methods, there are still many application challenges.

Geolocation assisted routing is also based on existing active or on-demand routing protocols. Through position prediction technology or GPS global positioning technology, the location of the target point is determined quickly. A routing algorithm based on location and topology information was proposed in literature [1]. This method borrowed the routing discovery mode of the on-demand routing protocol DSR and added location information during route discovery to reduce data flooding. This method was simple to operate but did not consider the problem of network routing holes. Literature [2] proposed a routing optimization algorithm for WMSN networks. Searching for wave crest nodes by sending detection packets and detoured the network voids. This method was feasible, but it was only applicable to stationary sensor networks with large limitations. Literature [3] proposed a network routing algorithm based on geographic location prediction. The algorithm estimated the possible location of the node at the next moment based on the ARIMA prediction model, eliminated the nodes that might be broken, and selects links that could be sent at higher rates. However, this method was relatively complicated, the operating cost was high and the burden of the network became larger as nodes move.

A greedy optimized routing algorithm based on QoS was proposed for the above problems. Simulation experiments showed that this method was obviously superior to the GPSR routing algorithm in terms of average 
end-to-end delay, average route hops, and network overhead, and was suitable for multi-UAV joint combat communication environment.

\section{QOS-BASED GREEDY OPTIMAL ROUTING ALGORITHM}

With regard to the optimization algorithm, the method first optimizes the path based on the network detour characteristics, and secondly, it needs a node selection criterion to determine whether the node performs detouring and ensures energy balance. The QoS-based greedy optimal routing algorithm is regarded as a new route establishment scheme applied to the UAV cluster network communication.

\section{The Optimization of Optimization Algorithm.}

Most geography-based routing protocols [4] generally use a greedy algorithm to find the path to the destination node. This method usually selects the shortest path at the fastest speed, but when the sending node finds no node which is closer to the destination node in its communication range, a routing hole [5] is generated.

\section{Routing Hole Type.}

The most common method for solving the routing hole problem is to planarize the network connection graph according to the distribution of the nodes $[6,7]$ and forward the data towards the nodes closer to the destination node until the greedy algorithm can be used again [8]. Because in the UAV network, the nodes move fast, the position changes frequently, and the holes are generated randomly and in various forms. This simple peripheral forwarding algorithm for routing discovery based on static topology information is no longer applicable and it requires a more Flexible way to complete the route.

\section{Routing Hole Solution.}

The existence of routing holes greatly increases the length of the path, so that more nodes are added to the path forwarding process, the protocol adopts an inverse optimization mechanism based on the existing paths and improves the data structure in the data transmission process, which is a good solution to this problem. This solution does not generate redundant packets nor increase latency.

\section{Packet Structure.}

In the conventional path optimization protocol, a hole boundary or an optimization path was usually found in the form of a probe packet. This would not only increase the network load but also make the delivery time of the packet longer. In Packet Replacement Routing Optimization Protocol (PSOR), by changing the structure of the data packet, the packet forwarding path was recorded and the data packet was sent on demand, ensuring low network overhead and low transmission delay, enabling the network to change the topology in real time, and then improving the stability of the network.

In order to achieve the above goals, we need to define a new data structure - Segment Cache. Table 1 shows the structure of the Segment Cache, where Segment Point records the node identified as a segment point in the path. Figure 1 shows the packaging form of Segment Cache.

TABLE 1. Segment Cache

\begin{tabular}{cc}
\hline Field names & Function \\
\hline Destination & Identify the destination node of the package \\
Segment Point & Record segment points \\
Prior Sending State & Record the forwarding status of the previous node \\
\hline
\end{tabular}




\begin{tabular}{|c|c|c|}
\hline $\begin{array}{c}\text { Destination } \\
(8 \text { bits })\end{array}$ & $\begin{array}{c}\text { Segment Point } \\
(8 \text { bits })\end{array}$ & $\begin{array}{c}\text { Prior Sending } \\
\text { State } \\
(8 \text { bits })\end{array}$ \\
\hline
\end{tabular}

FIG. 1. Packaging Form of Segment Cache

Add the defined data structure to the data packet structure. The modified packet structure is shown in Table 2 . The SC Array field is an array of Segment Cache type. This field is used to record the information of segment points during the forwarding process. Figure 2 shows the encapsulation of the packet.

TABLE 2. Packet structure

\begin{tabular}{cc}
\hline Field names & Function \\
\hline Type & Identify the type of the packet \\
Source & Identify the source node of the packet \\
*Destination & Use segment points as secondary destinations \\
Sequence & Determine whether the packet is received repeatedly \\
SC Array & Record segmentation status \\
Data & Forwarding information \\
\hline
\end{tabular}

\begin{tabular}{|c|c|c|c|}
\hline $\begin{array}{c}\text { Type } \\
(8 \text { bits })\end{array}$ & $\begin{array}{c}\text { Source } \\
\text { (8 bits) }\end{array}$ & $\begin{array}{c}\text { *Destination } \\
\text { ( } 8 \text { bits })\end{array}$ & $\begin{array}{c}\text { Sequence } \\
(8 \text { bits })\end{array}$ \\
\hline \multicolumn{4}{|c|}{ SC Array } \\
(24 bits)
\end{tabular}

FIG. 2. Encapsulation of the Packet

\section{Reverse Optimization Mechanism.}

After receiving the data packet, the destination node read the information contained in the data packet. To ensure that the subsequent data could be transmitted with shorter delay and less energy loss, the destination node optimizes the path in the boundary forwarding state. As shown in Figure 3, node S first initiates a data transmission to node D. According to the greedy forwarding algorithm, when the data reaches node A, it encounters a routing hole. Then, the forwarding mode changes to the boundary forwarding algorithm and reaches point $\mathrm{B}$. The forwarding mode reverts to the greedy forwarding. When data is sent to node $\mathrm{C}$, routing hole is encountered again. The data packet is forwarded to the E node according to the boundary forwarding algorithm, and then forwarded by the $\mathrm{E}$ to the $\mathrm{D}$ node according to the greedy algorithm. During the forwarding process, the packet marks the node which changes the forwarding mode from the boundary forwarding to the greedy forwarding as a segment point (eg. B and E) in the forward path and writes it in the SC Array field.

In the reverse optimization, the destination node $\mathrm{D}$ first reads the segment point in the data packet, sends it back to the node $\mathrm{E}$ in the greedy forwarding way. Then, the node $\mathrm{E}$ finds the next nearest segment node $\mathrm{B}$ from all the segment points and initiates a greedy forwarding process. If a new routing hole is generated due to node mobility during the forwarding process, the updated path and status are also recorded in the data packet. The same operation is performed from $\mathrm{B}$ to $\mathrm{S}$ and will not be described again.

The optimized path also reserveg the path optimization function during the data transmission. When the intermediate node failed due to the node's movement, it could be discovered and optimized in time. 


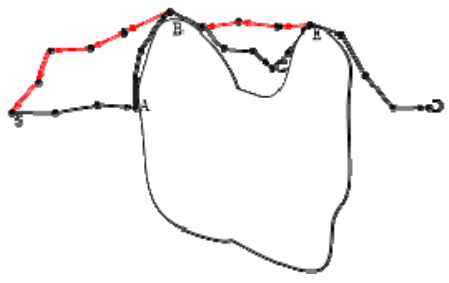

FIG. 3. Path Optimization Process

\section{Improvement of Node Selection Criterion.}

\section{The Traditional Greedy Algorithm for Node Selection.}

The greedy algorithm only selected the next hop node according to the position information of the neighbor nodes in the communication distance range with some certain simple rules. And it usually sacrificed a part of transmission performance to meet a single transmission requirement. For example, in order to reduce the transmission interference between nodes, more and more nodes were used as relays, thereby increasing the end-toend delay.

\section{QoS-based Node Selection Criterion.}

In the field of communications, energy consumption came mainly from data transmission. Avoiding low-energy nodes to send packets as much as possible was the main method to improve the detection capability.

In order to reduce the frequent changes in the topology caused by the link failure and the network energy consumption caused by the continuous update of routing information, it was necessary to ensure the stability of the topology. It need to consider selecting a more stable link as a route. This article defines the link stability as follows:

Definition 1 Link stability:

$$
L_{S N}=\left(1-\left(\mu \frac{d_{S N}-\min \left(d_{S N}, D\right)}{R-\min \left(d_{S N}, D\right)}+v \frac{V_{S N}}{V_{\max }}\right)\right)^{2}
$$

Among them, $\mu+v=1, \mathrm{~S}$ and $\mathrm{N}$ are respectively the sending node and the receiving node, $d_{S N}$ is the distance between the sending node and the receiving node, $\mathrm{D}$ is the minimum communication distance between nodes, $V_{S N}$ is the relative moving speed between nodes, $V_{\max }$ is the maximum moving speed of the node.

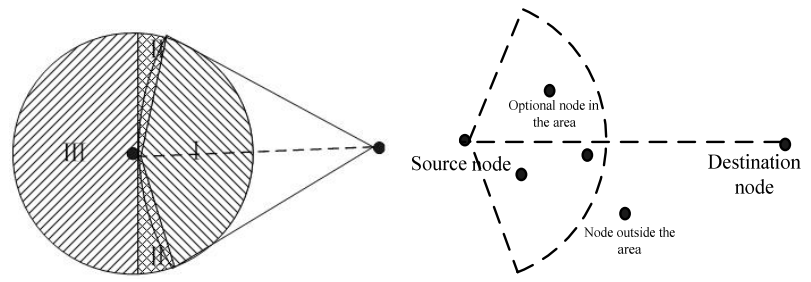

FIG. 4. Node Selection Range

As shown in Figure 4, the transmission range of the node is divided into three parts. Taking the connection line between the source node and the destination node as the axis direction and taking $\alpha$ angles on both sides of the axis as the selection area. In this area, the Euclidean distance between the receiving node and the destination node is less than the Euclidean distance between the sending node and the destination node. The selected receiving node is the best advantage of the I region. From the angle of $2 \alpha$ to the two sides continue to expand to 180 degrees, the upper 
and lower parts form the II region together. There are still some nodes in the area that satisfy I region's conditions, and these nodes need to be considered when selecting nodes. The remaining part of the node's transmission range constitutes the III region. When neither the I region nor the II region can select a node that meets the requirements, it is necessary to select a boundary forwarding node in the III region. At this point, the advantages of space transmission are no longer the main criteria, and energy balance is particularly important [9].

So, we got the formula of the node criterion:

$$
\begin{gathered}
N(i)=\beta L_{S N}+(1-\beta) E_{\text {res }} / E_{\text {cap }} \\
\begin{cases}\beta=0.6 & 0 \leq \theta<2 \alpha \\
\beta=0.5 & 2 \alpha \leq \theta<\pi \\
\beta=0.4 & \pi \leq \theta \leq 2 \pi\end{cases}
\end{gathered}
$$

Among them, $N(\mathrm{i})$ is the selection criterion of the node, and the point with the largest criterion value in the selectable area is selected as the next hop node. $\beta$ is the correction factor, according to the value of the node's location. $E_{r e s}$ is the remaining energy of the node and $E_{c a p}$ is the total energy of the node.

\section{OPTIMIZED ROUTING PROTOCOL}

\section{Exchange and Maintenance of Node Position Information}

With the development of positioning technology, satellite positioning systems such as GPS or Beidou can be installed on the UAV nodes to obtain the location of nodes. First, the new node acquired a network notification by broadcasting a HELLO packet to the surrounding nodes. The new node completed the network access of the node by sending a network access request to the central control node and receiving a reply response from the central control node. After the node completed network access, it immediately sent a SOP packet carrying the location information of this node, and it is eligible to receive and forward SOP packets of other nodes. Nodes could not receive SOP packets from other nodes before they completed network access. By receiving SOP packets from other nodes, the node gradually collected geographic location information of all nodes in the whole network and established a node position information table.

The key to the above network access plan was that each node maintained a next-hop node to the currently control node at all times. The next-hop node to the control node might become invalid at any time due to the node movement and the switching of the control node. Therefore, every node should detect the effectiveness of its next hop node every other SOP cycle. Once it found that its next-hop node to the control node had become invalid, such as the next-hop node had moved beyond its own single-hop communication distance, or the current central control node had switched, this node would immediately find a new effective next-hop node according to the current position of the control node.

\section{Forward Route Discovery}

According to the geography information of the known nodes, the path selection method based on the directional angle restriction and the optimal node selection was adopted in the discovery process of forward routing. The specific algorithm steps were as follows:

(1) When the source node had a packet to send, set the packet type to 1, the Priority Sending State to 0 . Take the source node and the destination node as the initial segmentation point. Go to step (2).

(2) The node first acquired the geography information of the destination node (or the secondary destination node). Next it calculated the distance from the destination node (or the secondary destination node) to the own node and judged whether it was within the single-hop communication range of the node. If so, the destination node (or the secondary destination node) was set as the next hop node and sent directly, otherwise it went to step (3). 
(3) According to the information in the node location information table, the line from the node to the destination node (or the secondary destination node) was taken as the axis, and the arc was drawn on both sides with the singlehop communication distance of the node. The angle was 2 a and determined a closed sector area.

(4) According to the node selection criterion formula in the protocol design, the optimal node was selected as the next hop from all the nodes contained in the sector area, and the contents of the Prior Sending State field were checked. If Prior Sending State $=0$, this node was inserted into the Segment Point field as a segmentation point and the packet was sent to the next hop node. Set Prior Sending State=1. If there is no node in the selected sector, go to step (5), otherwise go to step (6).

(5) The node expanded the selection range of the next hop node to 180 degrees. According to the modified node selection criterion formula, the suboptimal hop node was selected from the two small sector areas as the next hop node, and the contents of the Prior Sending State field were checked. If Prior Sending State $=0$, then this node was inserted as a segmentation point into the Segment Point field, and the packet was sent to the next hop node. Set Prior Sending State=1. If there is no node in the sector area, enter the boundary forwarding mode to perform step (7); otherwise, perform step (6) directly.

(6) The node received the data packet from the last hop and determined if it is the destination node (or the secondary destination node), and if so, it received the packet; otherwise, it look up whether the packet had been received based on the source node and the serial number in the record. If the packet is received, the packet is not forwarded and should be deleted. If the packet is not received, repeat step (2) of the route discovery process.

(7) Set the packet type Type=2. The node that entered the boundary forwarding also selected the local optimal node from the sector area as the next hop node according to the revised node selection criterion formula and sent it. Set the Prior Sending State to 0. Go to step (6).

In order to reduce the adverse effect on the stability of the link caused by the node movement, when selecting the next hop node, the single hop communication distance could be appropriately reduced. For example, if the singlehop communication distance is $250 \mathrm{~km}$, the distance may be defined as $200 \mathrm{~km}$ when the fan-shaped area is determined. The theory could prove that there was no closed-loop phenomenon in the sending process.

\section{Reverse Path Segmented Optimization}

According to the reverse optimization of mechanism, the received packet record path could be optimized. The specific steps were as follows:

(1) The destination node read the packet type after receiving the packet and set the source node as the destination node. This node was set as the source node. If Type $=1$, the path is the optimal path. When no data was requested, this node no longer sent packets. If Type $=2$, the node read the SC Array field, judged the segmentation point and wrote backwards into the packet segmentation field. Set Type $=1$. Use the first segmentation point as the secondary destination node. The data was forwarded to the next hop in the form of forward route discovery. Go to step (2).

(2) The node received the data packet and determined whether this node was the destination node. If so, this node receives the packet. Otherwise, the node continued to determine whether the current node was a secondary destination node. If so, the next segment point in the SC Array field was used to take the place of the current node as the secondary destination node and forwarded the data in the forward route discovery mode. Otherwise, the packet was sent to the secondary destination node.

The route discovery process of reverse segmented optimization is similar to that of forward route discovery and will not be described here.

\section{SIMULATION ANALYSIS}

This article uses OPNET [10] software for simulation. In order to verify the optimized performance of the algorithm, this paper chose the GPSR [11] routing protocol which was the most common in geography routing protocol, and the Boundary Optional Perimeter Forwarding (BOPF) algorithm which improved the routing hole problem [12]. The experimental results were compared from the average end-to-end delay, the average hop number and the network load under the different node. Simulation parameters are as follows: 
TABLE 3. Main parameters of simulation

\begin{tabular}{cc}
\hline Parameter & Value \\
\hline Size & $10 \mathrm{~km} * 10 \mathrm{~km}$ \\
Number of nodes & $60,70,80,90,100,110$ \\
Coverage of Communication & $1 \mathrm{~km}$ \\
MAC protocol & SPMA \\
Speed & $200 \mathrm{~m} / \mathrm{s}$ \\
\hline
\end{tabular}

Figure 5 shows the trends of average end-to-end delays at different node densities for the PSOR, GPSR, and BOPF protocols. It can be seen from the figure that the PSOR protocol is significantly better than the GPSR protocol, and there are also certain advantages compared with the BOPF protocol. This was because the PSOR protocol could quickly find a local optimal path to reach the destination node and could ensure that the path was globally optimal in the subsequent data transmission process through the optimization of path. The GPSR protocol maintained the same path after a transmission process. This not only resulted in routing redundancy, but also increased holds due to the excessive consumption of node energy and increased the delay of path bypass. Although the BOPF protocol could also find a local optimal path, it was necessary to calculate the angle between the two nodes and the baseline after the hole was encountered. In addition, the protocol was less adaptable when the number of nodes was small and the hole boundary was complex. With the increase of the number of nodes, the end-to-end delay present an upward trend. The reason was that there were more nodes involved in the forwarding and the transmission delay increased. However, the PSOR protocol was more adaptable to the extension of network scale than the GPSR protocol and the BOPF protocol.

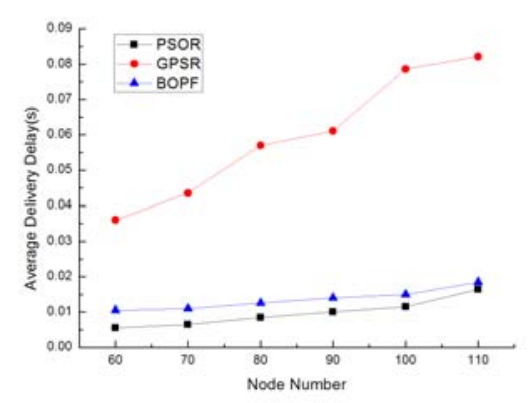

FIG. 5. Average Delivery Delay

Figure 6 shows the trend of the average route hops for the PSOR, GPSR, and BOPF protocols at different node densities. It can be seen that compared with the GPSR protocol, the average number of hops for the PSOR protocol has been saved by $28.12 \%$, which is $8.5 \%$ lower than the BOPF protocol. This is because the PSOR routing protocol can optimize the emerging network holes in time and select the optimal node through the criteria of node selection to avoid wasting energy. With the increase in node density, the number of nodes in the forwarding path also increased, the optimization effect is more apparent. The GPSR protocol selected the transmission node through a single standard and could not optimize the bypass nodes. The problem of node waste was outstanding. While the BOPF protocol does route optimization for routing holes, it cannot adapt to the high-speed moving environment of the nodes, and it responds slowly to changes in the topology, which also leads to excessive consumption of special nodes. 


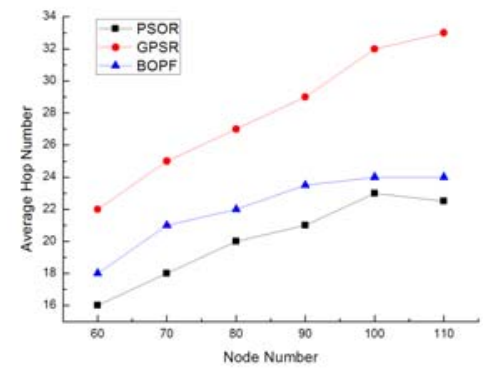

FIG. 6. Average Hop Number

Figure 7 shows the network load changes at different node densities for the PSOR, GPSR, and BOPF protocols. It can be seen that the PSOR protocol has little difference in network load compared with the GPSR protocol and the BOPF protocol because the main network load comes from the exchange of location information in the network. When the node density is small, the PSOR protocol increases the load slightly due to the introduction of the path information to the path optimization. However, with the increase of network node density, the optimization effect on the path is obvious. Therefore, the transmission of excess node and the network load decreases. The BOPF protocol needs to return more landmark data when the number of nodes is small and the boundary of hole is complex, thus increasing the routing overhead.

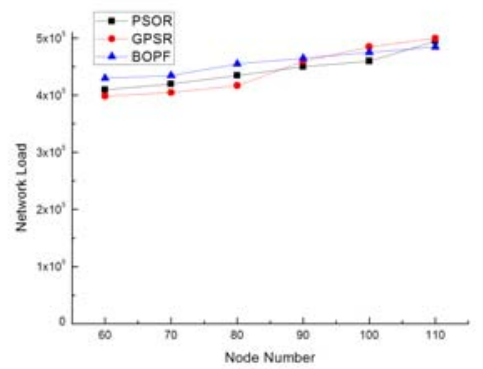

FIG. 7. Network Load

\section{SUMMARY}

This paper proposed a solution algorithm for routing holes in location routing protocols. The node selection mechanism in the sub-region ensured that nodes could choose the next hop at a faster speed. Considering the characteristics of UAV, the comprehensive criterion formula of node selection was introduced to ensure that the path selection satisfied the energy balance and enhances the scalability and the service life of the network. Simulation experiments showed that the reverse optimization mechanism of the PSOR protocol could solve the routing problem of the network very well. Although it caused a slight increase in the network load, it was still within an acceptable range. And the PSOR protocol made obvious optimization to the network performance. The protocol could meet the communication needs of UAV cluster operations.

\section{REFERENCES}

1. Cao Yinglie, Xie Shengli, Qiu Hongyan. Hybrid routing algorithm based on location and topology information in MANET[J]. Journal of South China University of Technology (Natural Science Edition). Vol.35(2007) No.1, p.74-78.

2. Tang Ruichun. HOGGH: A Routing Optimization Algorithm for WMSN Networks[J]. Journal of Ocean Unive rsity of China.Vol.40(2010) No.11, p.123-128. 
3. Sha Yi. An Ad Hoc Network Routing Algorithm Based on Geographic Location Prediction[J]. Miniature Micr ocomputer System.Vol.36(2015) No.3, p.493-496.

4. Y. B. Ko and N. H. Vaidya. Location-Aided Routing (LAR) in Mobile Ad Hoc Networks, 1998[C]. Proc. Conf. Mobile Computing MOBICOM,1998, p.66-75.

5. Q. Fang,J. Gao,and L. J. Guibas. Locating and Bypassing Routing Holes in Sensor Networks, Hong Kong, 2004[C]. Proc. IEEE INFOCOM 2004. Hong Kong,2004.

6. K GARBRIEL, R SOKAL.A new statistical approach to geographic variation analysis[J]. Systematic Zoology. 1969, p.259-278.

7. G TOUSSAINT. The relative neighborhood graph of a finite planar set[J]. Pattern Recogni. 1980, p.261-268.

8. Chen D,Varshney P K. A survey of void handling techniques or geographic routing in wireless networks[J]. IEEE of Communications Surveys and Tutorials. Vol.9(2007) No. 1, p. 50-67.

9. B. Ramakrishnan, M. Selvi, R. Bhagavath Nishanth. Efficiency measure of routing protocols in vehicular ad hoc network using freeway mobility model[J]. Wireless Net. Vol.23(2017), p.323-333.

10. Chen Min. OPNET Network Simulation[M]. Beijing: Tsinghua University Press,2004.

11. B KARP, H KUNG. GPSR: Greedy Perimeter Stateless Routing for Wireless Networks, USA, 2000 [C]. Proceedings of the Sixth Annual ACM International Conference on Mobile Computing and Networking (MobiCom'00). USA, 2000, p.243-254.

12. Qi Qian.Research on Routing Algorithm for Mobile Ad Hoc Networks Based on Location Information[D]. Harbin: Harbin Institute of Technology,2013. 